\title{
A sensitive search for the emission of a neutral particle in the decay of the first excited state in ${ }^{16} \mathrm{O}$.
}

\section{P. Sona}

Dipartimento di Fisica and Istituto Nazionale di Fisica Nucleare,Florence,Italy

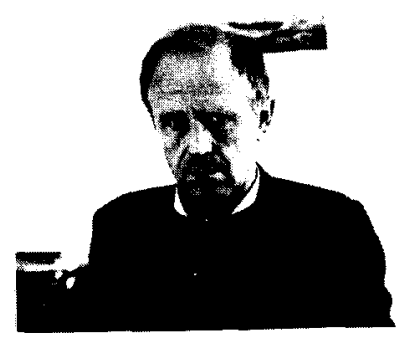

M.Bini, T.Fazzini, A.Giannatiempo, G.Poggi and N. Taccetti

Dipartimento di Fisica and Istituto Nazionale di Fisica Nucleare, Florence, Italy.

\section{K.Elsener}

CERN Geneve, Switzerland.

P.G.Hansen and E.Uggerh $\varnothing \mathrm{j}$

Institute of Physics, University of Aarhus, Denmark.

\section{ABSTRACT}

A search by invariant mass spectroscopy has been performed for a possible weak branch in the decay of the first excited state of ${ }^{16} \mathrm{O}$ proceeding via the emission of a neutral particle decaying into $\mathrm{e}^{+} e^{-}$pairs. Upper limits of $.02 \%$ to $.1 \%$ (90\% confidence level) for this branch have been derived for a particle mass in the 1.5-3.1 $\mathrm{MeV}$ range. 


\section{Introduction}

Triggered by the recent spectacular findings at GSI in Darmstadt ${ }^{1)}$ about which we heard many contributions at this workshop, several groups have searched for the production of neutral exotic objects (named $\mathrm{X}$ in the following) decaying into $e^{+} e^{-}$pairs. Among the numerous attempts in this direction, the most recent ones aimed at detecting the possible emission of an $\mathrm{X}$ in the deexcitation of a nuclear level by an experimental set-up suitable for the case of a short-lived $\left(\tau \leq 10^{-11} s\right.$ ) particle decaying into $e^{+} e^{-}$pairs ${ }^{2,3)}$. None of this experiments, however, made use of the invariant mass spectroscopy which is the most sensitive, albeit difficult, way to look for the presence of a new particle. We decided therefore to look again, by applying invariant mass spectroscopy, at the decay of the first excited $J^{\pi}=0^{+}$state at $6.05 \mathrm{MeV}$ in ${ }^{16} \mathrm{O}$ which proceeds via internal pair conversion (IPC) with a lifetime of $(96 \pm 7) \times 10^{-12} \mathrm{~s}^{4}$. This long lifetime is due to the fact that the single gamma decay to the $J^{\pi}=0^{+}$ground state is completely forbidden thus giving a chance for a possible weak branch via $\mathrm{X}$ emission to show up. The problem at hand is to distinguish,

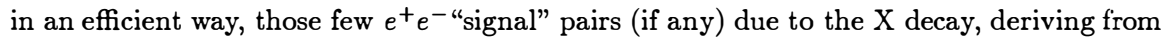
the "background" pairs due to the normal IPC decay. From the kinematics of the X decay it follows that the measurement of the pair opening angle $\Theta_{0}$ is already quite efficient in discriminating against background pairs since the latter have a smooth correlation function ( of the $1+\cos \Theta_{0}$ type), while the signal pairs have a correlation which, for any given mass $\mathrm{M}_{0}$ of the $\mathrm{X}$ from, say, $1.3 \mathrm{MeV}$ up to about $1.8 \mathrm{MeV}$, is strongly peaked at a particular opening angle. This state of affairs (already pointed out a decade ago ${ }^{5}$ ) ) is shown in Fig.1. It is also apparent from Fig. 1 that for higher mass values the peak would rapidly disappear and the measurement of the opening angle should be supplemented by the measurement of the energy of one member of the pair so as to reconstruct the invariant mass, unless one is willing to discard most of the data by restricting the analysis to a narrow energy window centered around the "symmetric"energy split of about $3 \mathrm{MeV}$. Further analysis shows that a rather crude energy measurement together with an accuracy of about $1.5^{\circ}$ (standard deviation) on $\Theta_{0}$ is sufficient to guarantee an invariant-mass resolution (FWHM) of about $120 \mathrm{keV}$. These considerations justify most of the features of the adopted experimental set-up.

\section{Experimental set-up and measurements}

The proton beam of the KN3000 Van der Graaff accelerator of the INFN in Firenze was used to populate the first excited state in ${ }^{16} \mathrm{O}$ via the reaction ${ }^{19} \mathrm{~F}(\mathrm{p}, \alpha){ }^{16} \mathrm{O}$. The target consisted of a $.3 \mathrm{mg} / \mathrm{cm}^{2}$ layer of $\mathrm{MgF}_{2}$ deposited on a $30 \mu \mathrm{g} / \mathrm{cm}^{2}$ carbon backing. Typical beam currents of $.2-.3 \mu \mathrm{A}$ were used. A beam energy of $1.73 \mathrm{MeV}$ was chosen to optimize the population ratio of the first excited state relative to that of nearby levels which could produce spurious pairs through external conversion of the deexciting $\gamma$ rays. The target was placed at the center of a cylindrical vacuum chamber having a radius of $160 \mathrm{~mm}$. A closely packed silicon telescope, consisting of a $25 \mathrm{~mm}^{2} x .3 \mathrm{~mm}$ detector followed by a 50 $\mathrm{mm}^{2} \times 2 \mathrm{~mm}$ detector, was placed inside the chamber with its front face at $130 \mathrm{~mm}$ from the target. A $50 \mu \mathrm{m}$ thick mylar foil was mounted close to the front face of the telescope to shield it from scattered protons and delta rays. The angular position of the telescope was controlled through an electromechanical transducer to a precision of $.3^{0}$. A standard ${ }^{6,7)}$ 
$10 \mathrm{~cm} \times 10 \mathrm{~cm}$ multiwire proportional chamber (MWPC) was placed outside the vacuum chamber behind a $120 \mu \mathrm{m}$ thick mylar window. The wire spacing in both the vertical and horizontal direction was $.11 \mathrm{~cm}$. The plane of vertical wires faced the target at a distance of $20 \mathrm{~cm}$. A NE102A scintillator $(12 \mathrm{~cm} \times 12 \mathrm{~cm}, 4 \mathrm{~cm}$ thick $)$ was placed behind the MWPC to measure the positron or electron energy. The resolution was measured to be $160 \mathrm{keV}$ at 1 $\mathrm{MeV}$ and found to scale as the square root of the energy. The acquisition system was based on a VME bus controlled by a Mac Intosh computer. The acquisition cycle was triggered by requiring a coincidence between the the telescope, the scintillator and the MWPC. The range of the pair opening angle covered in a single exposure was about $23^{\circ}$. To explore the invariant mass range between 1.5 and $3.1 \mathrm{MeV}, 4$ runs were required corresponding to different angular settings of the telescope, for a total of 800 hours of beam time.

\section{Analysis and results}

In order to formulate a quantitative statement about the value of the branch, one has to know the shape and detection efficiency for both the signal and background spectra. To this end a detailed Monte Carlo simulation was carried out taking into account several effects: a) production depth of the pair in the target.b) scattering in the target. c) scattering in the mylar window of the vacuum chamber. d) measured response function of the MWPC properly corrected for geometrical effects and double hit events. e) energy lost in crossing the MWPC. f) additional energy release in the scintillator due to (single) Compton scattering of annihilation quanta. g) finite size of the telescope. In Fig.2 is reported the experimental invariant mass spectrum obtained in a particular run together with the corresponding simulated spectrum. Both spectra were obtained by setting a window from 1.5 to $3.5 \mathrm{MeV}$ on the energy spectrum of the scintillator and deducing the energy of the other member of the pair from the available decay energy $(=6.05 \mathrm{MeV})$. The agreement appears quite satisfactory (as confirmed by the $\chi^{2}$ value obtained by introducing only an overall normalization constant) provided one excludes the wings of the spectra which are critically affected by complicated scattering effects which cannot be reliably reproduced in the Monte Carlo simulation. To give an idea of the sensitivity of the present experiment, an example of the "signal" spectrum expected for a $0.5 \%$ branch via the emission of an $\mathrm{X}$ with a mass of $2.8 \mathrm{MeV}$ is also reported in Fig.2. The final analysis of the data was performed using a minimum $\chi^{2}$ procedure, by fitting, for each run and each assumed mass of the $\mathrm{X}$, a linear combination of the simulated "signal" and "background" spectra to the experimental spectrum. No evidence has been found for the presence of a branch, and we quote upper limits, (at the $90 \%$ confidence level), in $100 \mathrm{keV}$ steps, for the mass range 1.5-3.1 MeV. The results are summarized in Fig 3. These limits can be translated into lower limits for the isoscalar coupling strength $\alpha_{n}$ of the $\mathrm{X}$ to nucleons, by using the theoretical expressions for the decay rates via $\mathrm{X}$ emission and via IPC ${ }^{8)}$. The theoretical branch $B_{t}$ is given by

$$
B_{t}=\frac{15 \cdot \pi \alpha_{n} \beta^{5}}{2 \cdot \alpha^{2}}
$$

where $\alpha$ is the fine structure constant and $\beta$ is the $\mathrm{X}$ velocity in c units. For example, by comparing $\mathrm{B}_{t}$ to the upper limit for a mass of $1.8 \mathrm{MeV}$, we find $\alpha_{n}<6.2 \cdot 10^{-10}$. This limit is about an order of magnitude smaller than that derivable from similar experiments ${ }^{2,3}$ ) 


\section{References}

(1) T. Cowan et al., Phys. Rev. Lett. 55 (1986) 444.

(2) M. J. Savage, B. W. Filippone and L. W. Mitchell, Phys. Rev. D37 (1988) 1134.

(3) F. W. N. de Boer et al., J. Phys. G: Nucl. Phys. 14 (1988) L131.

(4) M. Birk, J. S. Sokolowski and Y. Wolfson, Nucl. Phys. A216 (1973) 217

(5) A. Hallin and F. P. Calaprice, Bull. Am. Phys. Soc. 24 (1979) 63

(6) R. Bouclier et al., Nucl. Inst. Meth. 88 (1970) 149.

( 7) F. Sauli, CERN Yellow report 1977/09.

(8) L. Resnick, M. K. Sundaresan and P. J. S. Watson, Phys. Rev. D8 (1973) 172.

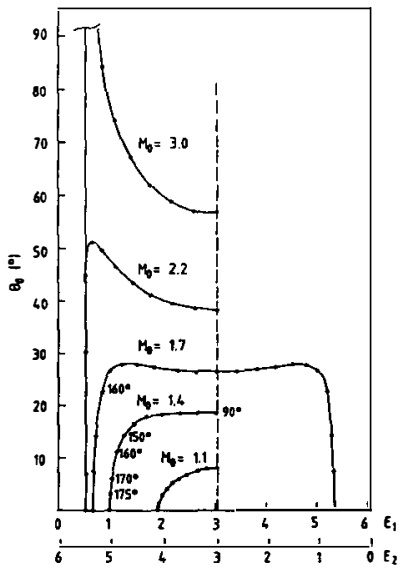

Fig. 1: Relation between the laboratory opening angle $\Theta_{0}$ and relativistic energies $E_{1}, E_{2}$ (in $\mathrm{MeV}$ ) of the $e^{+} e^{-}$pair. Each curve is labeled by the mass $M_{0}$ (in $\mathrm{MeV}$ ) of the $\mathrm{X}$. The points indicate values of the $\mathrm{CM}$ angle relative to the direction of the X. The decay has been assumed isotropic.

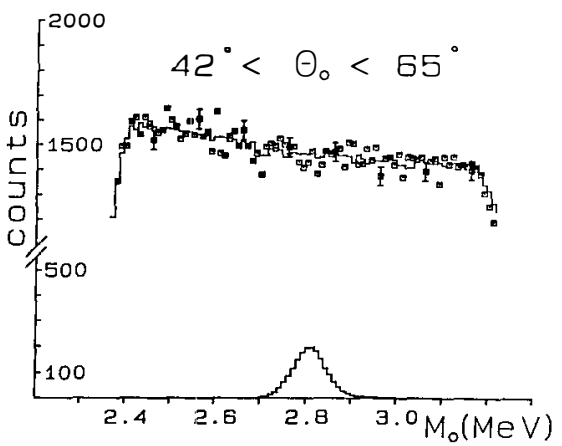

Fig. 2 Invariant-mass spectrum for the run in which the angle between the telescope and the center of the MWPC (as seen from the target) is $53^{\circ}$. The squares indicate experimental data while the histogram represents the normalized Monte Carlo spectrum for the normal IPC decay. The simulated spectrum for an hypothetical

$0.5 \%$ branch via emission of an $\mathrm{X}$ with a mass of $2.8 \mathrm{MeV}$ is shown in the lower part.

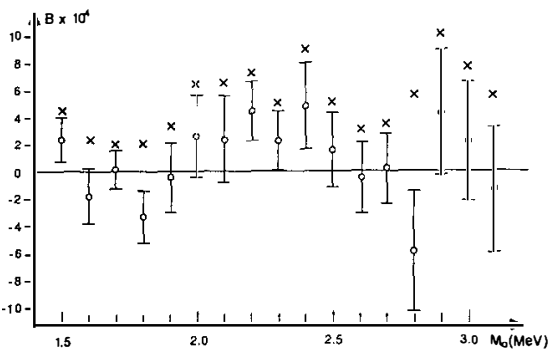

Fig. 3 Branching ratio $\mathrm{B}$, deduced by the procedure indicated in the text, as a function of the invariant-mass $M_{0}$ of the X. Upper limits at the $90 \%$ confidence level are given by crosses. 